\title{
Energy Controlled Reporting for Industrial Monitoring Wireless Sensor Networks
}

\author{
Geoff V. Merrett, Nick R. Harris, Bashir M. Al-Hashimi, Neil M. White \\ School of Electronics and Computer Science, University of Southampton, UK \\ \{gm04r, nrh, bmah, nmw\}@ecs.soton.ac.uk
}

\begin{abstract}
This paper proposes a technique to extend the lifetime of a wireless sensor network through a combination of energy management and information control. Each sensor node locally decides its own network involvement as a result of balancing available energy resources with the information content of each packet. The information content is ascertained through a system of rules which describe prospective events in the sensed environment. These rules specify when reporting should occur, and the importance of each packet. While energy management and information content have been individually considered elsewhere, our technique utilizes a combination of both to incur greater benefits. Results obtained from a simulation depicting an industrial Wireless Sensor Network (WSN) monitoring a water pumping station have shown that a considerable increase in lifetime and connectivity can be obtained. In addition, when coupled with energy harvesting, our technique permits sustained operation.
\end{abstract}

\section{INTRODUCTION}

A Wireless Sensor Network (WSN) typically consists of a number of small, inexpensive, locally powered sensor nodes that communicate detected events wirelessly via multihop routing [1]. WSNs are continuing to receive an increasing research interest, largely due to the wide range of suitable applications, including environmental monitoring [2], military [3], and education [4]. A key research area is concerned with extending the limited network lifetime, inherent to the sensor nodes [1]. This has been attempted from various approaches, including energy-aware routing algorithms [5], MAC protocols and adaptive sampling [6].

ReInForM [7] uses knowledge about the channel error, number of hops, and data importance to send packets along multiple routes. The number of different routes used depends on the importance of the packet (implemented elsewhere), thus linking packet reliability with information content. In Hull et al [8], a bandwidth management method is described, using a rule system to prioritize outgoing data packets depending on their derived importance (not considering energy management). In Jain et al [9], a Dual Kalman Filter architecture is used to reduce the bandwidth by only communicating data that cannot be predicted by the base station. This however requires a pre-selected, powerful base station, and complex mathematical operations at each sensor node. TinyDB [10] is an acquisitioned query processing system for sensor networks. The system allows a base station to extract relevant data from the network of sensors by interrogating it with SQL-like queries. Priorities (not linked to the queries) can be added to outgoing packets to overcome congestion. Power management exists by optimizing queries and adjusting reporting rates. TinyDB is a general solution, requiring comparatively high overheads.

In this paper, we propose a localized technique for extending the lifetime referred to as IDEALS/RMR (Information manageD Energy Aware aLgorithm for Sensor networks with Rule Managed Reporting). The lifetime is extended through the synergy of information control and energy management, which results in the node's behavior being determined both by their local energy state and the importance of events occurring in their environment. We believe that this union has not been considered before. The basic principles of IDEALS were introduced in Merrett et al. [11], where it was simulated under static conditions. The aim of this paper is to continue this work by quantifying the information content of sensed data through RMR, and by performing an in-depth simulation of IDEALS/RMR in a realistic scenario with intermittent energy harvesting.

\section{IDEALS/RMR}

The concept of IDEALS/RMR is that a node with a high energy reserve acts for the good of the network by participating in routing all packets that come to it, and by generating its own packets from all locally detected events. However, a node with a near-depleted energy reserve acts selfishly, by only generating or forwarding important packets. Through this, IDEALS extends the network lifetime for important data, through the possible loss of more trivial data. The computational costs introduced are low, as only simple mathematical operations are required.

\section{A. $R M R$}

The purpose of RMR is to determine if an event worth reporting has occurred, and how important such an event is. A range of methods exist for deciding when a node should report that an event has occurred. The simplest method is to report periodically, every $t$ minutes (meaning that packets are transmitted even when the sensed parameter has not significantly changed). The second option is a querying approach, where the base station instigates data transfer by

This research is funded in part by the Engineering and Physical Science Research Council (EPSRC) under grant number EP/D042917/1. 
requesting data from a subset of the nodes [10]. The third method is for the sensor node to decide locally when events should be reported. This is the method that RMR uses.

Before deploying a network, the designer creates a set of rules describing differing events that can be detected in the sensed environment. These rules include threshold rules (the sensed value crosses a preset value), differential rules (the change in the sensed value is larger or smaller than a preset value), feature rules (a pattern or feature is noticed in the sensed value), periodic rules (periodically, every $t$ time), and routine rules (a packet of that importance or higher has not been sent for a period of time). For examples of these rules, see table 1 . The rules are also assigned a message priority (MP), relating to the importance of the event. A high message priority (MP1) relates to an important event (e.g. a large temperature change). Conversely, a low message priority (MP5) relates to a low importance event (e.g. no temperature change). Intermediate priorities MP2-MP4 are allocated to events whose information content lies between the two extremes. On receiving sensor data, if any rules are fulfilled, RMR generates a packet with the associated MP.

\section{B. IDEALS}

IDEALS continuously assigns the node a power priority (PP) based on the state of the energy reserve and harvesting environment. Nodes with high energy reserves are allocated a high power priority (PP5), while near depleted energy resources are allocated a low power priority (PP1). Intermediate priorities PP2-PP4 relate to the power levels which lie between these extremes. When a packet is to be sent or forwarded, IDEALS compares the MP and PP. A packet will be sent if the PP $\geq$ MP. Therefore, as the residual energy drops, packets will be selectively discarded in order of their information content. For example, if the battery is full (PP5), packets with any information content (MP1MP5) will be transmitted. However, if the battery is low (PP1), only packets with a high information content (MP1) will be transmitted. A fraction of the energy is allocated to PP0 to maintain an energy store for power management, during which no sensing or communications takes place.

\section{SimULATING IDEALS/RMR}

This paper considers the realistic, simulated scenario of a WSN used to monitor the temperature of 20 randomly organized pumps in a water pumping station (organized as shown in fig. 1). In the simulated scenario, each pump operates once daily for a random period of time, with an ambient temperature of $25^{\circ} \mathrm{C}$, and an operating temperature of $50^{\circ} \mathrm{C}[12]$. Sensors are duty cycled, inspecting the pump every five minutes. The simulation is run over a period of five days. During pump operation, mechanical vibrations occur locally, allowing $0.1 \mathrm{~mW}$ of harvested power to supplement the nodes' batteries (calculated from a pump vibrating at 25 milli ' $\mathbf{g}$ ' - where $\mathbf{g}$ is $\left.9.81 \mathrm{~m} / \mathrm{s}^{2}[12]\right)$. While the pump is inactive, no energy is harvested. The energy figures used equate to the nodes being powered from a $1.2 \mathrm{~F}$ super-capacitor, and consuming around $35 \mathrm{~mW}$ to transmit.

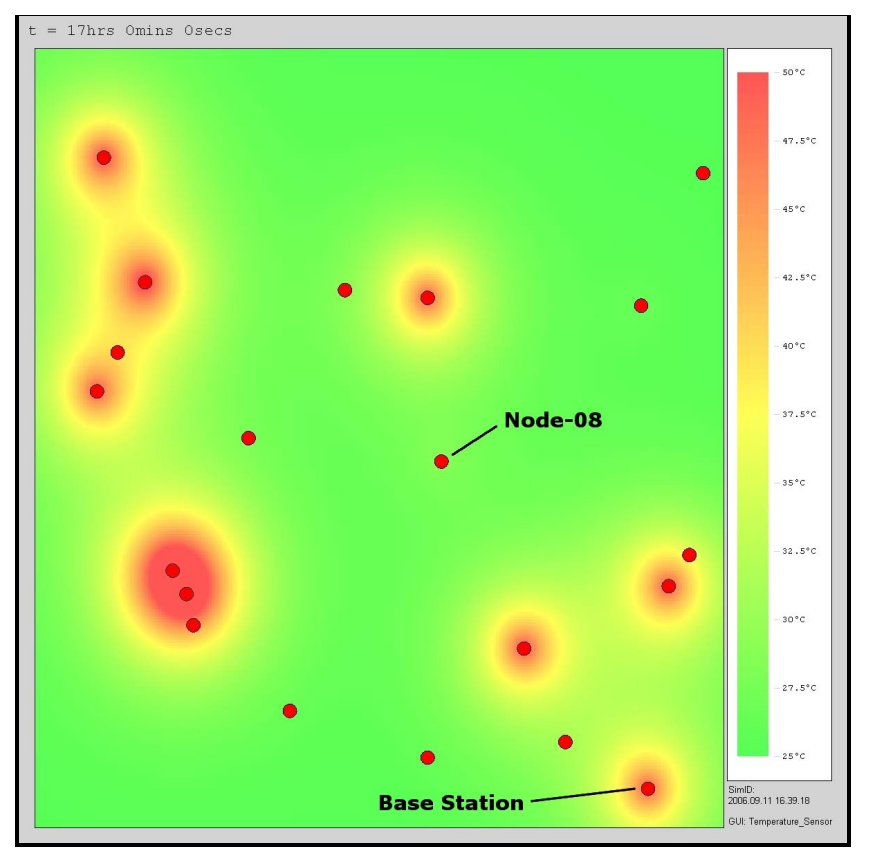

Figure 1. Temperature profile during simulation of the network in WSNim, where small circles represent sensor nodes (attached to pumps).

The network was simulated using an in-house simulator (WSNsim) developed as part of this research (fig. 1). The use of a custom simulator (as opposed to numerous available simulators [13]) enabled the structured, unproblematic integration and customizability of IDEALS/RMR into the simulator. WSNsim uses the layered system architecture of the Unified Framework [14] to represent communications, sensing and energy management with equal precedence and structure (see fig. 2.). The routing algorithm currently implemented in WSNsim is flooding, due to its inherent simplicity. We believe that advantages proposed by IDEALS/RMR can be applied to a wide range of different routing algorithms, and this is a direction of future investigation. A low-power receive radio transceiver is used to limit effects of packet flooding. Energy harvesting can be modeled in WSNsim using a mathematical representation, tabulated data, or a combination of both.

When IDEALS/RMR is active, reporting rules are defined as shown in table 1 . These rules were created by inspecting the normal operating conditions of the pump, and anticipating various possible faults of varying importance.

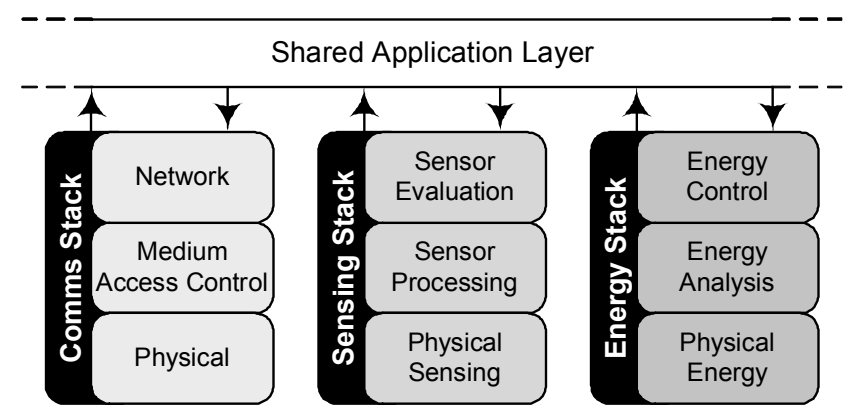

Figure 2. The unified stack (created in accordance with the Unified Framework [14]) that is incorporated into WSNsim. 
While successful operation relies on the careful selection of parameters such as reporting rules and MP/PP thresholds, we believe that it should be a simple process to a designer having knowledge of the target application.

TABLE I. RMR RULES USED IN THE SIMULATIONS

\begin{tabular}{|c|c|c|c|}
\hline Rule Type & Value & Priority & Primary Use \\
\hline \multirow{4}{*}{ Threshold } & $37.5^{\circ} \mathrm{C}$ & 4 & Detect pump on/off operation \\
\cline { 2 - 4 } & $20^{\circ} \mathrm{C}$ & 2 & Detect pump too cool \\
\cline { 2 - 4 } & $10^{\circ} \mathrm{C}$ & 1 & Detect pump very cool \\
\cline { 2 - 4 } & $55^{\circ} \mathrm{C}$ & 2 & Detect pump too hot \\
\cline { 2 - 4 } & $70^{\circ} \mathrm{C}$ & 1 & Detect pump very hot \\
\hline \multirow{3}{*}{ Differential } & $2^{\circ} \mathrm{C}$ & 2 & Detect pump on/off operation \\
\cline { 2 - 4 } & $10^{\circ} \mathrm{C}$ & 1 & Detect sensor failures \\
\hline \multirow{3}{*}{ Routine } & $2 \mathrm{hrs}$ & 5 & \multirow{2}{*}{ Provide routine reports } \\
\cline { 2 - 4 } & $4 \mathrm{hrs}$ & 3 & \\
\cline { 2 - 3 } & $6 \mathrm{hrs}$ & 1 & \multicolumn{2}{|c}{} \\
\hline
\end{tabular}

Two different sets of data were created and simulated for the pumping station: normal conditions (the pumping station operating with no faults), and fault conditions (the pumping station operating with numerous faults). For each set of data, four simulations were conducted: a) traditional (no IDEALS/RMR or vibration harvesting), b) vibration harvesting (no IDEALS/RMR), c) IDEALS/RMR (no vibration harvesting), and d) IDEALS/RMR (with vibration harvesting). For the node specific simulation results shown in this section, node-08 (highlighted in fig. 1) is monitored, as it plays a critical role in network communications, and has the average number of neighboring nodes (four). By looking at an individual sensor node as opposed to the average of all nodes, we can inspect how the localized algorithms operate.

\section{Simulation Results}

\section{A. Normal Operating Conditions}

The normal operation data represent the case where all pumps and nodes are operating correctly. The data were generated using random pump on times and durations, with exponential growth and decay. These data were simulated to investigate the lifetime extension that is possible through the use of IDEALS/RMR, under normal conditions.

1) Node Energy Levels: Fig. 3 shows the energy levels of node- 08 for the duration of the simulations, where ' 1 ' means that the node's energy reserve is full, while ' 0 ' means it is depleted. It can be seen that in simulation ' $a$ ', node- 08 depletes its energy reserve after around nine hours, as it is sending a packet every five minutes. When energy harvesting is enabled (simulation ' $b$ '), the node receives sporadic energy increases. In simulation ' $c$ ', the energy level of node-08 does not deplete in a linear fashion. This is because of the threshold/priority system that IDEALS introduces, and is explained in greater detail in the controlled simulations of [11]. RMR has provided a significant extension in the lifetime as it has differentiated between events and redundant data. Finally, in simulation 'd' IDEALS/RMR has been enhanced by energy harvesting, and suggests the possibility of sustained operation.

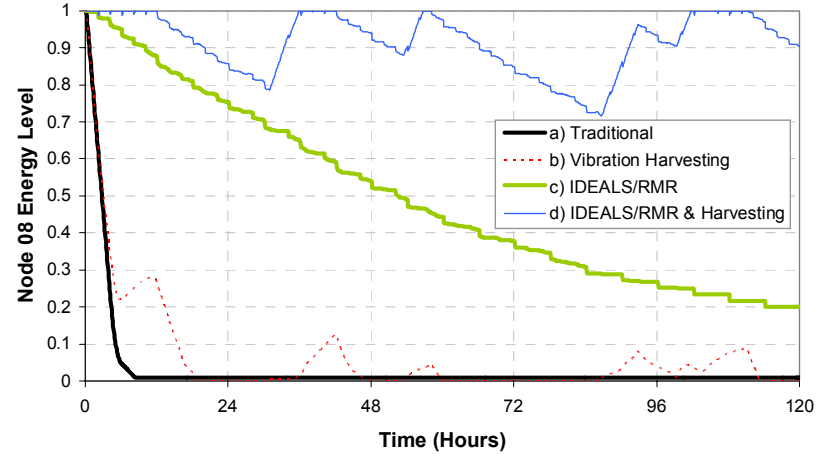

Figure 3. Node energy levels for the normal operating conditions

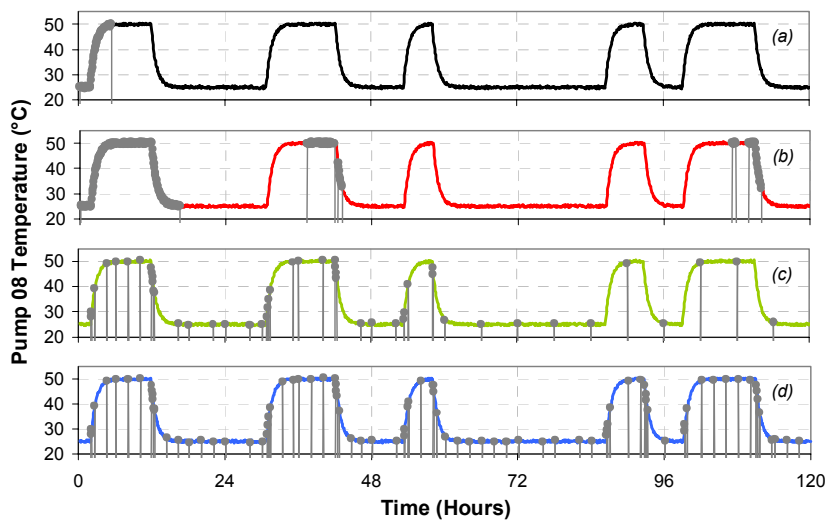

Figure 4. Message success for normal operating conditions, a) traditional, b) vibration harvesting, c) IDEALS/RMR, d) IDEALS/RMR + harvesting.

2) Packet Success: Fig. 4 shows the packet success statistics for node-08. This graph shows the packets (depicted by grey dots) that were transmitted from node-08 and successfully received by the base station. Packets that were sent by the node, but not received by the base station (due to a lack of network connectivity) are not shown. In simulation 'a' packets are generated every five minutes for 5 hours 25 minutes, after which no more packets are received by the base station. This short lifetime is due to the sending of regular (often redundant) data. Simulation 'b' sees packets received sporadically for the duration of the simulation due to intermittent energy harvesting. The sporadic nature does not provide a reliable and dependable network - for example, the pump turning on and off during days three and four are not reported. At the beginning of the simulation c), node- 08 has a full energy reserve, and so any event is reported (this can be seen as many packets are sent during the first 12 hours). However, towards the end of the simulation, the energy in the node's reserve has significantly reduced, and the node is operating in power priority 1 (PP1). Because of this, only the most important packets (of MP1) are transmitted and successfully received by the base station. In simulation ' $d$ ', an abundance of packets have been successfully received for the entire duration of the simulation. IDEALS/RMR has balanced the node's energy resources and the information content of packets to convey an accurate representation of the pump to the base station. 
TABLE II. FAULTS IN THE 'FAULT CONDITIONS' SimULATION

\begin{tabular}{|c|c|c|c|}
\hline Node/Pump & Fault & Seen As & Time (hrs) \\
\hline Node-03 & Sensor failure & Reads $0{ }^{\circ} \mathrm{C}$ & $21-49$ \\
\hline Node-06 & Sensor drift & Reading decreases & $25-49$ \\
\hline Node-08 & Sensor failure & Reads $0{ }^{\circ} \mathrm{C}$ & $58-71$ \\
\hline Pump-10 & Bearing failure & Temp. rise of $8^{\circ} \mathrm{C}$ & $32-55$ \\
\hline Node-13 & Sensor drift & Reading increases & $72-90$ \\
\hline All pumps & Fire & Temp. rise of $50^{\circ} \mathrm{C}$ & $100-110$ \\
\hline
\end{tabular}

\section{B. Fault Conditions}

The fault data is used for the case where multiple pump and sensor faults occur during the simulation. Faults (shown in table 2) were added to a basic set of data (generated in the same way as normal conditions) to determine how the network reacts to an influx of packets, and if RMR accurately portrays the events to the base station.

1) Node Energy Levels: Fig. 5 shows the energy levels of node- 08 for the duration of the fault simulation. The simulations featuring IDEALS/RMR demonstrate that the nodes adapt energy usage depending on occurring events, and so a greater energy depletion occurs around areas of faults to ensure that the base station is notified. In particular, the overheating pumps have caused a 50\% drop in energy level in simulation ' $\mathrm{d}$ ' because every node in the network is flooding a packet reporting this fault. This expenditure could be limited through in network data fusion. Following network degradation, IDEALS/RMR controls the recovery.

2) Packet Success: Fig. 6 shows the packet success statistics for node-08. It can be seen that simulation ' $a$ ' misses both faults as the node quickly depletes its energy

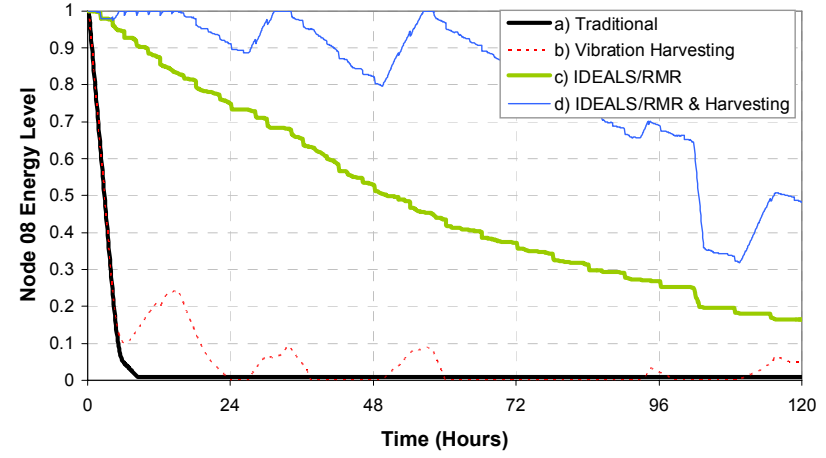

Figure 5. Node energy levels for the fault conditions

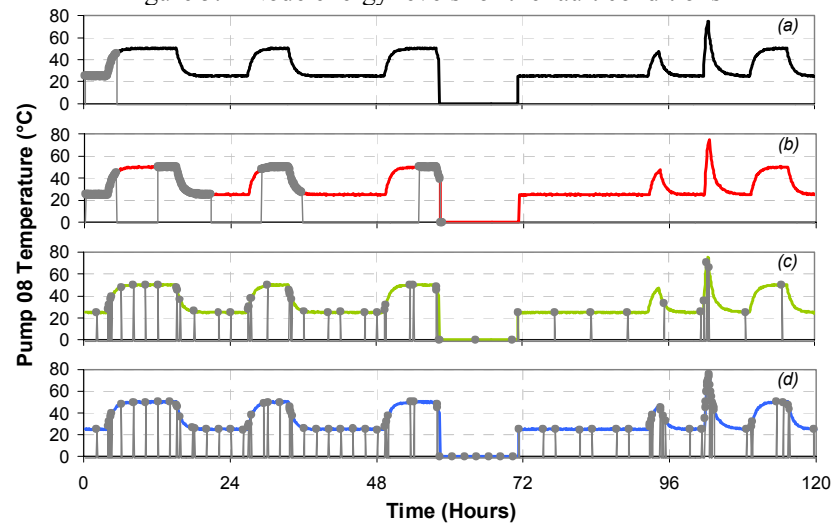

Figure 6. Message success for fault conditions, a) traditional, b) vibration harvesting, c) IDEALS/RMR, d) IDEALS/RMR + harvesting. reserve. In ' $b$ ', the ability to report events is random, dependant on sporadic energy increases from harvesting. In simulations ' $c$ ' and ' $d$ ', IDEALS/RMR has managed the network to allow both faults to be reported, while harvesting in ' $d$ ' has permitted reporting with greater resolution.

\section{CONCLUSIONS}

In this paper, we have introduced IDEALS/RMR, which operates locally upon a combination of information control (assessed through a custom set of rules describing the sensed environment) and energy management (balancing residual energy and energy harvesting with packet importance). We believe this has not been considered before. The results obtained from simulating a scenario where a WSN is used for temperature monitoring in a water pumping station have highlighted the ability of IDEALS/RMR to control the degradation of the network (providing an extension in the lifetime), and to report specific faults. IDEALS/RMR has provided a significant increase in the lifetime, and when coupled with energy harvesting, permits perpetual operation.

\section{REFERENCES}

[1] I.F. Akyildiz, W. Su, Y. Sankarasubramaniam, and E. Cayirci, "Wireless sensor networks: A survey," Computer Networks, vol. 38, pp. 393-422, 2002.

[2] A. Mainwaring, J. Polastre, R. Szewczyk, D. Culler, and J. Anderson, "Wireless sensor networks for habitat monitoring," Proc. 1st Workshop Wireless Sensor Networks and Applications, Atlanta, GA, Sep. 2002

[3] G. Simon, M. Maróti, Á. Lédeczi, G. Balogh, B. Kusy, A. Nádas et al., "Sensor network-based countersniper system," Proc. Conf. Embedded Networked Sensor Systems, Baltimore, MD, pp. 1-12, Nov. 2004

[4] M. Srivastava, R. Muntz, and M. Potkonjak, "Smart kindergarten: Sensor-based wireless networks for smart developmental problemsolving environments," Proc. 7th Ann. Int'l Conf Mobile Computing and Networking, Rome, Italy, pp. 132-138, Jul. 2001

[5] K. Akkaya and M. Younis, "A survey on routing protocols for wireless sensor networks," Ad Hoc Networks, vol. 3, pp. 325-349, 2005.

[6] V. Raghunathan, S. Ganeriwal, and M. Srivastava, "Emerging techniques for long lived wireless sensor networks," Communications Magazine, vol. 44, pp. 108-114, 2006.

[7] B. Deb, S. Bhatnagar, and B. Nath, "Reinform: Reliable information forwarding using multiple paths in sensor networks," Proc. 28th Conf. Local Computer Networks, Bonn, Germany, pp. 406-415, Oct. 2003

[8] B. Hull, K. Jamieson, and H. Balakrishnan, "Bandwidth management in wireless sensor networks," Proc. Int'l Conf. Embedded Networked Sensor Systems, Los Angeles, CA, pp. 306-307, Nov. 2003

[9] A. Jain, E.Y. Chang, and Y.-F. Wang, "Adaptive stream resource management using kalman filters," Proc. Int'l Conf. on Management of Data, (SIGMOD'04), Paris, France, pp. 11-22, Jun. 2004

[10] S.R. Madden, M.J. Franklin, J.M. Hellerstein, and W. Hong, "Tinydb: An acquisitional query processing system for sensor networks," $A C M$ Transactions on Database Systems, vol. 30, pp. 122-173, 2005.

[11] G.V. Merrett, B.M. Al-Hashimi, N.M. White, and N.R. Harris, "Information managed wireless sensor networks with energy aware nodes," Proc. 2005 NSTI Nanotechnology Conf. and Trade Show (NanoTech'05), Anaheim, CA, vol. 3, pp. 367-370

[12] Perpetuum ltd, http://www.perpetuum.com/; last accessed Aug. 2006.

[13] The network simulator - ns2 (ISI, University of Southern California), www.isi.edu/nsnam/ns; last accessed Jun. 2005

[14] G.V. Merrett, A.S. Weddell, N.R. Harris, N.M. White, and B.M. AlHashimi, "The unified framework for sensor networks: A systems approach," unpublished, University of Southampton, Technical Report, Sept. 2006, http://eprints.ecs. soton.ac.uk/12955/ 